PROCEEDINGS OF THE

AMERICAN MATHEMATICAL SOCIETY

Volume 135, Number 9, September 2007, Pages 3021-3028

S 0002-9939(06)08872-1

Article electronically published on November 29, 2006

\title{
COMPLETE MANIFOLDS WITH NONNEGATIVE CURVATURE OPERATOR
}

\author{
LEI NI AND BAOQIANG WU
}

(Communicated by Jon G. Wolfson)

\begin{abstract}
In this short note, as a simple application of the strong result proved recently by Böhm and Wilking, we give a classification on closed manifolds with 2-nonnegative curvature operator. Moreover, by the new invariant cone constructions of Böhm and Wilking, we show that any complete Riemannian manifold (with dimension $\geq 3$ ) whose curvature operator is bounded and satisfies the pinching condition $R \geq \delta \frac{\operatorname{tr}(R)}{2 n(n-1)} \mathrm{I}>0$, for some $\delta>0$, must be compact. This provides an intrinsic analogue of a result of Hamilton on convex hypersurfaces.
\end{abstract}

\section{INTRODUCTION}

Let $(M, g)$ be a Riemannian manifold. The curvature operator of $(M, g)$ lies in the subspace $S_{B}^{2}\left(\bigwedge^{2} T M\right)$ of $S^{2}\left(\bigwedge^{2} T M\right)$ cut out by the Bianchi identity. The decomposition $S_{B}^{2}\left(\bigwedge^{2} T M\right)=\langle\mathrm{I}\rangle \oplus\left\langle\operatorname{Ric}_{0}\right\rangle \oplus\langle\mathrm{W}\rangle$ splits the space of algebraic curvature operators into $O(n)$-invariant orthogonal irreducible subspaces. For an orthonormal basis $\phi_{\alpha}\left(\right.$ say $\left.\phi_{\alpha}=e_{i} \wedge e_{j}\right)$ of $\bigwedge^{2} T M$ (which can be identified with $s o(n)$ ), the Lie bracket is given in terms of

$$
\left[\phi_{\alpha}, \phi_{\beta}\right]=c_{\alpha \beta \gamma} \phi_{\gamma}
$$

It is easy to check, by simple linear algebra, that $\langle[\phi, \psi], \omega\rangle=-\langle[\omega, \psi], \phi\rangle$. Here $\langle A, B\rangle=-\frac{1}{2} \operatorname{tr}(A B)$. This immediately implies that $c_{\alpha \beta \gamma}$ is anti-symmetric. If $A, B \in S^{2}\left(\bigwedge^{2} T M\right)$ one can define

$$
(A \# B)_{\alpha \beta}=\frac{1}{2} c_{\alpha \gamma \eta} c_{\beta \delta \theta} A_{\gamma \delta} B_{\eta \theta} .
$$

It is easy to see that $A \# B$ is symmetric too. Also from the anti-symmetry of $c_{\alpha \beta \gamma}$, $A \# B=B \# A$.

In [BW], a remarkable algebraic identity was proved on how a linear transformation of $S_{B}^{2}\left(\bigwedge^{2} T M\right)$ changes the quadratic form $Q(R)=R^{2}+R^{\#}$. Böhm and Wilking then constructed a continuous pinching family of invariant closed convex cones. Using this construction they confirmed a conjecture of Hamilton stating that on a compact manifold the normalized Ricci flow evolves a Riemannian metric with 2-positive curvature operator to a limit metric with constant sectional curvature.

Received by the editors June 22, 2006 and, in revised form, August 16, 2006.

2000 Mathematics Subject Classification. Primary 58J35.

The first author was supported in part by NSF Grants and an Alfred P. Sloan Fellowship. 
Hence it gives a complete topological classification of compact manifolds with positive 2-positive curvature operator. In this short note, based on the strong result and the techniques of [BW], we give the classification for manifolds with 2-nonnegative curvature operators and an application of their invariant cone constructions to the compactness of Riemannian manifolds with pinching curvature operator.

\section{A STRONG MAXIMUM PRINCIPLE}

Let $(M, g(t))$ be a complete solution to Ricci flow such that there exists a constant $A$ and the curvature tensor of $g(t)$ satisfies $\left|R_{i j k l}\right|^{2}(x, t) \leq A$, for all $(x, t) \in M \times$ $[0, T]$. In [H1], Hamilton proved that under the evolving normal frame the curvature tensor satisfies the following evolution equation.

Proposition 2.1 (Hamilton).

$$
\left(\frac{\partial}{\partial t}-\Delta\right) R=2\left(R^{2}+R^{\#}\right)
$$

where $R^{\#}=R \# R$.

The following was observed for compact manifolds in [Chen, H3]. We spell out the argument for the noncompact case for the sake of the completeness.

Proposition 2.2. The convex cone of the 2-nonnegative curvature operator is preserved under the Ricci flow.

Proof. Let I be the identity of $S_{B}^{2}\left(\bigwedge^{2} T M\right)$, which can be identified with the induced metric on $\bigwedge^{2} T M$ (as a section of $\left.\bigwedge^{2} T M \otimes \bigwedge^{2} T M\right)$. We also denote the identity map of $T M$ by id. With respect to the evolving normal frame we have that $\nabla \mathrm{I}=0$ and $\frac{\partial}{\partial t} \mathrm{I}=0$. Let $\psi(x, t)>0$ be the fast growth function constructed in Lemma 1.1 of [NT1] satisfying $\frac{\partial}{\partial t} \psi-\Delta \psi \geq C_{1} \psi$. Here $C_{1}$ can be chosen as arbitrarily large as we wish. We shall consider $\tilde{R}=R+\epsilon \psi \mathrm{I}$ and show that $\tilde{R}$ is 2-positive for every (sufficiently small) $\epsilon$. If not, by the boundedness of $R$ and growth of $\psi$ we know that it can only fail somewhere finite. Assume that $t_{0}$ is the first time $\tilde{R}$ fails to be 2-positive and it happens at some point $x_{0}$. Also assume an orthonormal basis $\omega_{\alpha}$ (it may not be in the form of $e_{i} \wedge e_{j}$ as $\phi_{\alpha}$ ) such that $\tilde{R}$ is diagonal (so is $R$ ) with eigenvalues $\mu_{1} \leq \mu_{2} \leq \cdots \leq \mu_{N}$, where $N=\frac{n(n-1)}{2}$. Parallel translate $\omega_{\alpha}$ to a neighborhood of $\left(x_{0}, t_{0}\right)$, and let $\tilde{R}_{\alpha \alpha}=\left\langle R\left(\omega_{\alpha}\right), \omega_{\alpha}\right\rangle$. Then at $\left(x_{0}, t_{0}\right)$ we have, by the maximum principle, that

$$
\begin{aligned}
0 \geq & \left(\frac{\partial}{\partial t}-\Delta\right)\left(\tilde{R}_{11}+\tilde{R}_{22}\right) \\
\geq & \left(R^{2}+R^{\#}\right)_{11}+\left(R^{2}+R^{\#}\right)_{22}+2 \epsilon C_{1} \psi \\
= & \left(\tilde{R}^{2}+\tilde{R}^{\#}\right)_{11}+\left(\tilde{R}^{2}+\tilde{R}^{\#}\right)_{22}+2 \epsilon C_{1} \psi \\
& +\left(R^{2}+R^{\#}-\tilde{R}^{2}-\tilde{R}^{\#}\right)_{11}+\left(R^{2}+R^{\#}-\tilde{R}^{2}-\tilde{R}^{\#}\right)_{22} \\
= & \mu_{1}^{2}+\mu_{2}^{2}+\sum\left(c_{1 \beta \gamma}^{2}+c_{2 \beta \gamma}^{2}\right) \mu_{\beta} \mu_{\gamma}+2 \epsilon C_{1} \psi \\
& -\epsilon \psi\left((2 \operatorname{Ric} \wedge \mathrm{id}+(n-1) \epsilon \psi \mathrm{I})_{11}+(2 \operatorname{Ric} \wedge \mathrm{id}+(n-1) \epsilon \psi \mathrm{I})_{22}\right) .
\end{aligned}
$$

In the previous equation we have used Lemma 2.1 of $[\mathrm{BW}$, which asserts that $R+R \# \mathrm{I}=\operatorname{Ric} \wedge \mathrm{id}$ (the use is not really necessary). Since $\mu_{1}+\mu_{2} \geq 0$ and $\mu_{\gamma} \geq 0$ 
for all $\gamma \geq 2$,

$\sum\left(c_{1 \beta \gamma}^{2}+c_{2 \beta \gamma}^{2}\right) \mu_{\beta} \mu_{\gamma}=2 \sum_{\gamma \geq 3}\left(c_{12 \gamma}^{2}+c_{21 \gamma}^{2}\right)\left(\mu_{1}+\mu_{2}\right) \mu_{\gamma}+\sum_{\beta, \gamma \geq 3}\left(c_{1 \beta \gamma}^{2}+c_{2 \beta \gamma}^{2}\right) \mu_{\beta} \mu_{\gamma} \geq 0$.

Note also that at $\left(x_{0}, t_{0}\right)$ we have that $\mu_{11}+\mu_{22}=0$, which implies that $R_{11}+R_{22}=$ $-2 \epsilon \psi$; then $2 \epsilon \psi\left(x_{0}, t_{0}\right) \leq 2 A$. Hence at $\left(x_{0}, t_{0}\right)$ we have that

$$
\begin{aligned}
0 & \geq\left(\frac{\partial}{\partial t}-\Delta\right)\left(\tilde{R}_{11}+\tilde{R}_{22}\right) \\
& \geq \mu_{1}^{2}+\mu_{2}^{2}+2 \epsilon C_{1} \psi-200 n A \epsilon \psi .
\end{aligned}
$$

This is a contradiction if we choose $C_{1}>100 n A$.

By choosing the barrier function more carefully as in [NT2, N1] (see for example Theorem 2.1 of [N1]), we can have the following strong maximum principle.

Corollary 2.3. Assume that $R(g(0))$ is 2-nonnegative and 2-positive somewhere. Then there exists $f(x, 0) \geq \frac{1}{2}\left(\mu_{1}+\mu_{2}\right)(x, 0)$, which satisfies $f(x, t)>0$ for $t>0$ and

$$
\left(\mu_{1}+\mu_{2}\right)(x, t) \geq f(x, t) .
$$

In particular, if $R(g(0))$ is 2-nonnegative and $\left(\mu_{1}+\mu_{2}\right)\left(x_{0}, t_{0}\right)=0$ for some $t_{0} \geq 0$, then $\left(\mu_{1}+\mu_{2}\right)(x, t) \equiv 0$ for all $(x, t)$ with $t \leq t_{0}$. Moreover, $\mu_{1}(x, t)=\mu_{2}(x, t)=0$ for all $(x, t)$ with $t \leq t_{0}$ and

$$
\mathcal{N}_{2}(x, t)=\operatorname{span}\left\{\omega_{1}, \omega_{2}\right\}
$$

is a distribution on $M$ which is invariant under the parallel translation. In particular, $(M, g(t))$, for $t \leq t_{0}$, has nonnegative curvature operator.

Proof. For the existence of $f$ and the lower estimate of $\mu_{1}+\mu_{2}$ please see [NT2], Theorem 2.1. If $\mu_{1}+\mu_{2}$ achieves its minimum at $\left(x_{0}, t_{0}\right)$, we can deduce from the lower estimate that $\left(\mu_{1}+\mu_{2}\right)(x, t) \equiv 0$ for all $(x, t)$ with $t<t_{0}$ (which implies that it also holds at $t=t_{0}$ by continuity). Apply (2.2) with $\epsilon=0$. We can conclude that $M_{i}(x, t) \equiv 0$ for $i=1,2$. Hence $R \geq 0$. Please see Corollary 2.1 of [NT2 for the details on the part that $\mathcal{N}_{2}(x, t)$ is parallel.

The above result implies the following classification of closed 2-nonnegative manifolds.

Corollary 2.4. Assume that $R(g(0))$ is 2-nonnegative. Then for $t>0$, either the curvature operator $R(g(t))$ is 2-positive, or $R(g(t)) \geq 0$. Hence Suppose $\left(M^{n}, g_{0}\right)$ is a closed Riemannian manifold with 2-nonnegative curvature operator. Let $\tilde{g}(t)$ be the lift to the universal cover $\tilde{M}$ of the solution $g(t)$ to the Ricci flow with $g(0)=g_{0}$. Then for any $t>0$ we have that either $\left(\tilde{M}^{n}, \tilde{g}(t)\right)$ is a closed manifold with 2-positive curvature operator or it is isometric to the product of the following:

(1) Euclidean space,

(2) closed symmetric space,

(3) closed Riemannian manifold with positive curvature operator,

(4) closed Kähler manifold with positive curvature operator on real $(1,1)$-forms.

Proof. It follows from the above corollary and Hamilton's classification result on the solutions with nonnegative curvature operator. See for example [CLN], Theorem 7.34 . 
Topologically, it is now known, by [BW], that simply-connected 2-positive manifolds is sphere, and the Kähler manifold in the last case is biholomorphic to the complex projective space by the earlier result of Mori-Siu-Yau. The fact that the curvature operator of the evolving metrics becomes either 2-positive or nonnegative has been observed in Chen. However, in Chen there is no clear statement of the strong maximum principle, namely Corollary 2.3, on which the observation relies. If evoking Theorem 2.3 of [N1, the splitting result on solutions of Ricci flow on a complete Riemannian manifold with nonnegative curvature operator, we can write a similar statement even when $M$ is not assume to be compact. However, in this case the Euclidean factor is only topological (not isometric). Also we do not know if a complete noncompact 2-positive Riemannian manifold is diffeomorphic to $\mathbb{R}^{n}$ or not.

\section{Manifolds With Pinched CURVAture}

In $[\mathrm{H2}$ Hamilton proved that any convex hypersurface (with dimension $\geq 3$ ) in Euclidean space with second fundamental form $h_{i j} \geq \delta \frac{\operatorname{tr}(h)}{n}$ id must be compact. In $\mathrm{CZ}$, using the pre-established estimates of $\mathrm{Hu}$ ] and [Sh2, Chen and Zhu proved the following weak version of the above-mentioned Hamilton's result in terms of curvature operators. Namely, they proved that if a complete Riemannian manifold $\left(M^{n}, g\right)$ with bounded and $\left(\epsilon, \delta_{n}\right)$-pinched curvature operator (with $n \geq 3$ ) in the sense that

$$
\left|R_{\mathrm{W}}\right|^{2}+\left|R_{\mathrm{Ric}_{0}}\right|^{2} \leq \delta_{n}(1-\epsilon)^{2}\left|R_{I}\right|^{2}=\delta_{n}(1-\epsilon)^{2} \frac{2}{n(n-1)} \operatorname{Scal}(R)^{2}
$$

for $\epsilon>0, \delta_{3}>0, \delta_{4}=\frac{1}{5}, \delta_{5}=\frac{1}{10}$ and $\delta_{n}=\frac{2}{(n-2)(n+1)}$, where $R_{\mathrm{W}}, R_{\mathrm{Ric}_{0}}$ and $R_{\mathrm{I}}$ denote the Weyl curvature tensor, traceless Ricci part and the scalar curvature part, then $M$ must be compact. The strong pinching condition was the one originally assumed in $[\mathrm{Hu}$ to obtain various estimates and the smooth convergence result. It was also shown in $\mathrm{Hu}$ ] that it implies that $R \geq \epsilon R_{I}$. In [N3] the first author showed that the above result of Chen-Zhu can be shown by the blow-up analysis of $\mathrm{H} 3$. and some nonexistence results on gradient steady and expanding solitons obtained in [N3. (The detailed proof of these nonexistence results was submitted to the 2004 ICCM proceedings a while ago. See also the book [CLN].) With the help of a family of invariant cones constructed in $\mathrm{BW}$, we can now prove the following general result.

Theorem 3.1. Let $\left(M^{n}, g_{0}\right)$ be a complete Riemannian manifold with $n \geq 3$. Assume that the curvature operator of $M$ is uniformly bounded $\left(\left|R_{i j k l}\right|(x) \leq A\right)$ and satisfies that

$$
R \geq \delta R_{\mathrm{I}}>0
$$

for some $\delta>0$. Then $\left(M, g_{0}\right)$ must be compact.

Recall that $R_{\mathrm{I}}=\frac{1}{n(n-1)} \operatorname{Scal}(R) \mathrm{I}$, where I is the identity of $S_{B}^{2}(s o(n))$. Hence $R_{\mathrm{I}}>0$ is equivalent to $\operatorname{Scal}(R)>0$, and the above result is a natural analogue of the Hamilton result for hypersurfaces.

Proof. Let $g(t)$ be the solution to the Ricci flow with initial metric $g_{0}$ constructed by [Sh1. First we show that if $M$ is noncompact, $g(t)$ can be extended to a longtime solution defined on $M \times[0, \infty)$. In order to do that we first show that for 
sufficiently small $b>0, R\left(g_{0}\right)$ lies inside the invariant cone constructed by Lemma 3.4 of $[\mathrm{BW}$. Recall from [BW] the linear transformation

$$
\mathrm{l}_{a, b}: R \rightarrow R+2(n-1) a R_{\mathrm{I}}+(n-2) b R_{\mathrm{Ric}_{0}} .
$$

More precisely

$$
\begin{aligned}
l_{a, b}(R) & =R+2 a \bar{\lambda} \mathrm{I}+2 b \mathrm{id} \wedge \operatorname{Ric}_{0}(R) \\
& =(1+2(n-1) a) R_{\mathrm{I}}+(1+(n-2) b) R_{\mathrm{Ric}_{0}}+R_{\mathrm{W}} .
\end{aligned}
$$

It is easy to see that $l_{a, b}\left(S_{B}^{2}(s o(n))\right) \subset S_{B}^{2}(s o(n))$ and is invertible if $a \neq-\frac{1}{2(n-1)}$ and $b \neq-\frac{1}{n-2}$. Using this linear map and Theorem 2 of [BW], a pinching family of invariant convex cones are constructed. In particular, as one step of the construction, it was shown that

Lemma 3.2 (Böhm-Wilking). For $b \in\left[0, \frac{1}{2}\right]$, let

$$
a=\frac{(n-2) b^{2}+2 b}{2+2(n-2) b^{2}} \text { and } p=\frac{(n-2) b^{2}}{1+(n-2) b^{2}} .
$$

Then the set $1_{a, b}(C(b))$, where

$$
C(b)=\left\{R \in S_{B}^{2}(s o(n)) \mid R \geq 0, \text { Ric } \geq p(b) \frac{\operatorname{tr}(\operatorname{Ric})}{n}\right\},
$$

is invariant under the vector fields $Q(R)$. In fact for $b \in\left(0, \frac{1}{2}\right]$ it is transverse to the boundary of the set at all boundary points $R \neq 0$.

We claim that there exists $b>0$ sufficiently small such that $R\left(g_{0}\right) \in 1_{a, b}(C(b))$, which is equivalent to the fact that $\mathrm{l}_{a, b}^{-1}\left(R\left(g_{0}\right)\right) \in C(b)$. For simplicity let $\tilde{R}=R\left(g_{0}\right)$, $\bar{\lambda}(\tilde{R})=\frac{\operatorname{Scal}(\tilde{R})}{n}$ and $\mathrm{l}=\mathrm{l}_{a, b}$. Direct computation shows that

$$
R:=1^{-1}(\tilde{R})=\tilde{R}_{\mathrm{W}}+\frac{1}{1+2(n-1) a} \tilde{R}_{\mathrm{I}}+\frac{1}{1+(n-2) b} \tilde{R}_{\mathrm{Ric}_{0}},
$$

which implies that

$$
\operatorname{Ric}\left(1^{-1}(\tilde{R})\right)=\frac{\bar{\lambda}(\tilde{R})}{1+2(n-1) a} \mathrm{id}+\frac{1}{1+(n-2) b} \operatorname{Ric}_{0}(\tilde{R})
$$

and

$$
\bar{\lambda}(R):=\frac{\operatorname{tr}\left(\operatorname{Ric}\left(1^{-1}(\tilde{R})\right)\right)}{n}=\bar{\lambda}(\tilde{R})\left(1-\frac{2(n-1) a}{1+2(n-1) a}\right) .
$$

Let $\tilde{\lambda}_{i}$ be the eigenvalues of $\operatorname{Ric}_{0}(\tilde{R})$. Then by the assumption (3.1) we have that

$$
\tilde{\lambda}_{i}+\bar{\lambda}(\tilde{R}) \geq \delta \bar{\lambda}(\tilde{R}) .
$$

Clearly we also have that

$$
\tilde{\lambda}_{i}+\bar{\lambda}(\tilde{R}) \leq n \bar{\lambda}(\tilde{R}) .
$$


We first check that $R$ satisfies the Ricci pinching condition. In fact if $\lambda_{i}$ are the eigenvalues of $\operatorname{Ric}_{0}(R)$, from the above formulae we have that

$$
\begin{aligned}
-\lambda_{i} & =-\frac{1}{1+(n-2) b} \tilde{\lambda}_{i} \\
& \leq \frac{1-\delta}{1+(n-2) b} \bar{\lambda}(\tilde{R}) \\
& =(1-\delta) \frac{1+2(n-1) a}{1+(n-2) b} \bar{\lambda}(R) .
\end{aligned}
$$

Then there exist $\delta_{1}>0$ and $b_{0}$ such that for all $b \in\left[0, b_{0}\right],-\lambda_{i} \leq\left(1-\delta_{1}\right) \bar{\lambda}(R)$. Then we can find $b_{1} \leq b_{0}$ such that for any $b \in\left[0, b_{1}\right], p(b) \leq \delta_{1}$. Hence $R=1_{a, b}^{-1}(\tilde{R})$ satisfies the pinching condition of $C(b)$. Now we check that $R=\mathrm{l}_{a, b}^{-1}(\tilde{R}) \geq 0$. Rewrite

$$
R=\tilde{R}-\frac{2(n-1) a}{1+2(n-1) a} \tilde{R}_{\mathrm{I}}-\frac{(n-2) b}{1+(n-2) b} \tilde{R}_{\mathrm{Ric}_{0}} .
$$

Noting that $a \rightarrow 0$ as $b \rightarrow 0$, we can find $b_{2}$ such that for any $b \in\left[0, b_{2}\right]$ we have that

$$
R \geq \frac{\delta}{2} \tilde{R}_{\mathrm{I}}-\frac{(n-2) b}{1+(n-2) b} \tilde{R}_{\mathrm{Ric}_{0}}
$$

But the eigenvalue (with respect to $e_{i} \wedge e_{j}$, where $\left\{e_{i}\right\}$ is a basis of $T M$ consisting of eigenvectors of $\left.\operatorname{Ric}_{0}(\tilde{R})\right)$ of the right-hand side operator can be computed as

$$
\frac{\delta}{2} \frac{\bar{\lambda}(\tilde{R})}{n-1}-\frac{b}{1+(n-2) b}\left(\tilde{\lambda}_{i}+\tilde{\lambda}_{j}\right) .
$$

Using (3.3), the above can be bounded from below by

$$
\bar{\lambda}(\tilde{R})\left(\frac{\delta}{2(n-1)}-\frac{2(n-1) b}{1+(n-2) b}\right)>0
$$

if $b$ is close to 0 . This shows that there exists $b_{3}>0$ such that for any $b \in\left(0, b_{3}\right]$, $R\left(g_{0}\right) \in \mathrm{l}_{a, b}(C(b))$.

Now the virtue of the proof of Theorem 5.1 in BW], along with the short time existence result of [Sh1], shows that the Ricci flow has long-time solution. Otherwise, by Theorem 16.2 of [H3], we would end up with a blow-up solution, which is nonflat, noncompact, but whose curvature operator $R=R_{\mathrm{I}}$. In view of Schur's theorem, this is a contradiction. Note that $R\left(g_{0}\right) \in \mathrm{l}_{a, b}(C(b))$ allows us to apply the generalized pinching set construction (Theorem 4.1) from [BW], and since the evolving metric has positive curvature operator and the manifold is assumed to be noncompact, the injectivity radius always has a lower bound in terms of the size of the curvature. All these ingredients allow us to perform Hamilton's blow-up analysis [H3] (Theorem 16.2).

We continue to show that the extra assumption that $M$ is noncompact will lead us to a contradiction by performing the singularity analysis of $[\mathrm{H} 3$ as $t \rightarrow \infty$. Note that for all $t, R(g(t))$ will stay in the cone $\mathrm{l}_{a, b}(C(b))$ for some fixed (but sufficiently small) $b$, by the tensor maximum principle, which can be verified in the same way as Proposition 2.2. Now we claim that the curvature of $g(t)$ satisfies that

$$
\text { Ric } \geq p \frac{\operatorname{tr}(\mathrm{Ric})}{n} \text { id }
$$


for some $p>0$. Let $R^{*}=R(g(t))$. First, by Lemma 3.2 we know that $R(g(t)) \in$ $l_{a, b}(C(b))$ for some fixed small $b$. Thus we can find $R \in C(b)$ such that $\mathrm{l}_{a, b}(R)=R^{*}$. Now let $\bar{\lambda}=\frac{\operatorname{tr}(\operatorname{Ric}(R))}{n}$ and $\lambda_{i}$ be the eigenvalues of $\operatorname{Ric}_{0}(R)$. By the assumption we have that $-\lambda_{i} \leq(1-p) \bar{\lambda}$. Now we compute the Ricci curvature and its trace for $R^{*}$. By the definition of $l_{a, b}$ we have that

$$
\operatorname{Ric}\left(R^{*}\right)=\operatorname{Ric}+2(n-1) a \bar{\lambda} \mathrm{id}+(n-2) b \operatorname{Ric}_{0}
$$

and

$$
\bar{\lambda}^{*}:=\frac{\operatorname{tr}\left(\operatorname{Ric}\left(R^{*}\right)\right)}{n}=\bar{\lambda}(1+2(n-1) a) .
$$

Letting $\lambda_{i}^{*}$ be the eigenvalue of $R^{*}$ we have that $\bar{\lambda}^{*}+\lambda_{i}^{*}=(1+2(n-1) a) \bar{\lambda}+$ $(1+(n-2) b) \lambda_{i}$. Therefore

$$
\begin{aligned}
-\lambda_{i}^{*} & =-(1+(n-2) b) \lambda_{i} \\
& \leq(1-p)(1+(n-2) b) \bar{\lambda} \\
& =(1-p) \frac{1+(n-2) b}{1+2(n-1) a} \bar{\lambda}^{*} \\
& \leq(1-p) \bar{\lambda}^{*}
\end{aligned}
$$

Here we have used the fact that $1+2(n-1) a=1+(n-1) \frac{(n-2) b^{2}+2 b}{1+(n-2) b}>1+(n-2) b$. This completes the proof of the claim (3.4).

Since for all $g(t)$, its Ricci curvature satisfies (3.4), this holds up on the blowdown/blow-up solutions, which after passing to its universal cover, are either a nonflat gradient steady soliton or a nonflat gradient expanding soliton, with nonnegative curvature operator, by results from $\mathrm{H3}$. (Theorem 16.5, Corollary 16.6). (See also [N2], Theorem 4.2 and [CZ].) This contradicts Corollary 3.1 of [N3].

\section{Discussions}

In [W], the topology of so-called $p$-positive manifolds was studied. In view of the result of Böhm-Wilking and a result of Schoen-Yau $[\mathrm{ScY}]$ stating that any noncompact complete 3 -manifold with positive Ricci curvature must be diffeomorphic to $\mathbb{R}^{3}$, it is reasonable to speculate that any noncompact complete Riemannian manifold $M$ with 2-positive curvature operator must be diffeomorphic to $\mathbb{R}^{n}$. Professor Wilking informed us that one can show that $M$ is aspherical with cyclic fundamental group. (See also $\mathrm{W}$.)

In N3] we speculated that any complete Riemannian manifolds with positive pinched Ricci curvature must be compact. Theorem 3.1 confirms it under a stronger assumption on the curvature operator. The problem in full generality still remains unsettled.

\section{ACKNowledgments}

Part of this paper was completed during the first author's visit to ETH, Zürich. He would like to thank ETH, especially Tom Ilmanen, for providing a stimulating environment and various discussions. He also held informal discussions on BW] with Ben Chow and Nolan Wallach. 


\section{REFERENCES}

[BW] C. Böhm and B. Wilking, Manifolds with positive curvature operator are space form, preprint.

[Chen] H. Chen, Pointwise $\frac{1}{4}$-pinched 4-manifolds, Ann. Global Anal. Geom. 9(1991), no. 2, 161-176. MR:1136125 (93b:53028)

[CZ] B.-L. Chen and X.-P. Zhu, Complete Riemannian manifolds with pointwise pinched curvature Invent. Math. 140(2000) no. 2, 423-452. MR1757002 (2001g:53118)

[CLN] B. Chow, P. Lu and L. Ni, Hamilton's Ricci flow, Graduate Studies in Mathematics, AMS Press, 2006, 608 pp.

[H1] R. Hamilton, Four-manifolds with positive curvature operator, J. Differenital. Geom. 24 (1986), 153-179. MR0862046 (87m:53055)

[H2] R. S. Hamilton, Convex hypersurfaces with pinched second fundamental form, Comm. Anal. Geom. 2(1994), no. 1, 167-172. MR1312684 (95m:53078)

[H3] R. S. Hamilton, Formation of singularities in the Ricci flow, Surveys in Differential Geom. 2(1995), 7-136. MR1375255 (97e:53075)

[Hu] G. Huisken, Ricci deformation of the metric on a Riemannian manifold, J. Differential Geom. 21(1985), no. 1, 47-62. MR0806701 (86k:53059)

[N1] L. Ni, Ricci flow and nonnegativity of sectional curvature, Math. Res. Lett. 11(2004), no. 5-6, 883-904. MR2106247 (2005m:53123)

[N2] L. Ni, Monotonicity and Kähler-Ricci flow. Geometric evolution equations, 149-165, Contemp. Math., 367, Amer. Math. Soc., Providence, RI, 2005. MR.2115758(2006a:53082)

[N3] L. Ni, Ancient solutions to Kähler-Ricci flow, Math. Res. Lett. 12(2005), no. 5-6, 633-653. MR 2189227 (2006i:53097)

[NT1] L. Ni and L.-F. Tam, Plurisubharmonic functions and the Kähler-Ricci flow, Amer. J. Math. 125(2003), no. 3, 623-654. MR1981036(2004c:53101)

[NT2] L. Ni and L.-F. Tam, Plurisubharmonic functions and the structure of complete Kähler manifolds with nonnegative curvature, J. Differential Geom. 64(2003), no. 3, 457-524. MR2032112 (2005a:32023)

[ScY] R. Schoen and S.-T. Yau, Complete three-dimensional manifolds with positive Ricci curvature and scalar curvature, Seminar on Differential Geometry, pp. 209-228, Ann. of Math. Stud., 102, Princeton Univ. Press, Princeton, N.J., 1982. MR0645740 (83k:53060)

[Sh1] W. X. Shi, Deforming the metric on complete Riemannian manifolds, J. Differential Geom. 30(1989), 223-301. MR.1001277 (90i:58202)

[Sh2] W. X. Shi, Ricci deformation of the metric on complete noncompact Riemannian manifolds, J. Differential Geom. 30(1989), 303-394. MR.1010165 (90f:53080)

[W] H. Wu, Manifolds of partially positive curvature, Indiana Univ. Math. J. 36(1987), no. 3, 525-548. MR 0905609 (88k:53068)

Department of Mathematics, University of California at San Diego, La Jolla, CalIFORNIA 92093

E-mail address: lni@math.ucsd.edu

Department of Mathematics, Xuzhou Normal University, Xuzhou, Jiangsu, People's Republic of China

E-mail address: wubaoqiang@xznu.edu.cn 